\title{
Cancer et psychisme : mythes et réalité
}

\section{Cancer and Psychism: Myths and Reality}

\section{Reich}

(C) Lavoisier SAS 2019

Les relations supposées entre psychisme et cancer ont depuis des décennies alimenté débats, discussions, controverses. Les études expérimentales, épidémiologiques et méta-analyses retrouvent des liens contradictoires concernant les liens entre stress et développement ultérieur d'un cancer.

Certaines études pratiquées sur l'animal ont pu quantifier des modifications biologiques liées au stress comme, entre autres, une baisse des facteurs immunitaires ou des modifications hormonales $[1,2]$. Toutefois, transposer de facto ces résultats chez l'humain reste un raccourci hasardeux. Les études épidémiologiques se heurtent à plusieurs difficultés méthodologiques, ce qui rend leurs résultats discordants, ce dont il est difficile de tirer des conclusions robustes. Parmi ces difficultés, on peut citer :

- le délai entre la mesure du stress ou des facteurs de stress supposés et la survenue d'un cancer avéré ;

- la prise en compte ou non de facteurs confondants potentiels comme, par exemple, des expositions professionnelles (amiante) ou des addictions reconnues comme cancérigènes (tabac, alcool), qui peuvent résulter soit du stress ou être transmis avec le stress ;

- le moment de mesure du stress soit après l'apparition du cancer, soit avant avec alors un risque de surestimation par les patients de leur niveau de stress antérieur.

Ces diverses difficultés seront abordées dans l'article de Cédric Lemogne, Sarah Dauchy et Michel Reich.

Dans ce numéro spécial, les divers contributeurs sollicités ont voulu apporter une réflexion personnelle et sans parti pris pour une meilleure compréhension de cette problématique.

Nous avons souhaité que le fil conducteur puisse reposer sur un discours scientifique, rationnel tout en préservant l'aspect « irrationnel » inclus dans les théories profanes émises par les patients et nécessaires à leur adaptation psychique. Le

\section{Reich ( $\square)$}

Équipe de psycho-oncologie, centre Oscar-Lambret, 3, rue Frédéric-Combemale, F-59020 Lille cedex, France e-mail : mreich@o-lambret.fr patient a besoin de «donner du sens à du non-sens », avait coutume de rappeler Alby et al. [3].

Les patients peuvent aussi parfois tester la part irrationnelle qui sommeille en tout praticien : "Docteur, vous pensez que c'est le stress qui a causé mon cancer? ». Par cette question, les patients ne nous renvoient-ils pas à nos propres interrogations faites d'identification, mais aussi de rejet quant à une théorie profane ? Celle-ci viendrait-elle mettre à mal le raisonnement médical technico-scientifique rationnel de tout praticien?

Rusniewski nous rappelle que «Si la médecine se situe $d u$ côté de la probabilité, de la rationalité, d'un savoir objectif, le malade et sa famille se situent dans le registre des croyances, de l'intuition, d'un savoir subjectif lié à l'histoire et à la personnalité de chacun » [4].

On ne peut donc faire abstraction de cela. C'est ce qu'essayera de démontrer Nicolas Penel à travers son expérience de clinicien confronté à la récurrence de cette question concernant les liens entre stress et cancer et comment l'appréhender durant les consultations médicales.

Yori Gidron analysera les liens entre stress et cancer par le biais d'une physiopathologie reposant entre autres sur l'hypothèse neuro-immunologique et le rôle de signaux inflammatoires médiés par le système immunitaire dans la genèse $\mathrm{du}$ processus de cancérogenèse et l'influence potentielle d'une hyperactivité du nerf vague. Il proposera certaines pistes de réflexion pour gérer le stress chez les patients atteints de cancer.

L'équipe de Michel Daher apportera un éclairage ethnographique quant aux mythes et tabous qui accompagnent la survenue d'un cancer chez des patients originaires du Bassin méditerranéen (pays du Maghreb et du Moyen-Orient). Elle soulignera l'importance pour les professionnels de santé de s'approprier ces mythes et tabous pour mieux les mettre en perspective dans la prise en charge singulière de ces populations atteintes de cancer.

Laura Estienne et ses collaborateurs aborderont sur un angle original les théories profanes quant à la survenue du cancer, et ce par le biais de la spiritualité. Par une étude qualitative s'appuyant sur une analyse lexicographique et 
thématique, ces auteurs essayeront de démontrer comment « un travail spirituel», effectué par les patients, peut leur permettre une certaine adaptation face à la maladie et d'y trouver un sens.

Bertrand Porro et son équipe démontreront l'importance d'une prise en charge psychologique précoce chez des femmes atteintes de cancer du sein et notamment pour accentuer la composante positive du stress et en particulier le PTG (post-traumatic growth). Ce PTG va permettre d'observer tous les changements psychologiques positifs et opérants chez ces patientes, leur permettant une meilleure adaptation face au cancer.

La survenue d'un cancer continuera à susciter tant chez les patients que chez les soignants des tentatives d'explications causales. Dans l'état actuel des connaissances, un cancer ne peut être relié directement au stress vécu antérieurement par les patients. Toutefois, le stress, facteur fragilisant de l'organisme humain et donc des défenses immunitaires, pourrait entrer dans le processus de la maladie. Un cancer déclaré restera source de stress pour les patients et ne doit donc pas être occulté dans la prise en charge oncologique, que ce soit par des techniques pharmacologiques ou psychothérapeutiques.

\section{Références}

1. Strange KS, Kerr LR, Andrews HN, et al (2000) Psychosocial stressors and mammary tumor growth: an animal model. Neurotoxicol Teratol 22:89-102

2. Moreno-Smith M, Lutgendorf SK, Sood AK (2010) Impact of stress on cancer metastasis. Future Oncol 6:1863-81

3. Alby N, Oppenheim D, Guex P, et al (1999) Cancer sens et nonsens. Le Journal des Psychologues 17:21-49

4. Rusniewski M (2012) Fin de vie et société : approche psychanalytique. In: Hirsch E (ed) Fins de vie, éthique et société. Érès, Toulouse, pp 57-61 